\title{
Criminologie
}

\section{La relativité culturelle de la victimisation. Quelques réflexions sur les problèmes et le potentiel de la victimologie comparée}

\section{Ezzat Fattah}

Volume 26, numéro 2, 1993

La criminologie comparée. Hommage à Denis Szabo

URI : https://id.erudit.org/iderudit/017342ar

DOI : https://doi.org/10.7202/017342ar

Aller au sommaire du numéro

Éditeur(s)

Les Presses de l'Université de Montréal

ISSN

0316-0041 (imprimé)

1492-1367 (numérique)

Découvrir la revue

Citer cet article

Fattah, E. (1993). La relativité culturelle de la victimisation. Quelques réflexions sur les problèmes et le potentiel de la victimologie comparée. Criminologie,

26(2), 121-136. https://doi.org/10.7202/017342ar
Résumé de l'article

The purpose of the paper is to present some reflexions on the cultural relativity of victimization. It argues against the widely held belief among victimologists that victimization can be universally defined. Like crime, victimization can be seen as a cultural construct. The paper presents many examples illustrating the cultural relativity of victimization. Children's work, child abuse and neglect, violence, abortion, sexual behavior etc. are given as examples to emphasize the variability in the definition from one society to another. The author concludes by proposing comparative research covering various aspects of victimization. 
LA RELATIVITÉ CULTURELLE DE LA VICTIMISATION

Quelques réflexions sur les problèmes et le potentiel de la victimologie comparée

Ezzat Fattah ${ }^{1}$

The purpose of the paper is to present some reflexions on the cultural relativity of victimization. It argues against the widely held belief among victimologists that victimization can be universally defined. Like crime, victimization can be seen as a cultural construct. The paper presents many examples illustrating the cultural relativity of victimization. Children's work, child abuse and neglect, violence, abortion, sexual behavior etc. are given as examples to emphasize the variability in the definition from one society to another. The author concludes by proposing comparative research covering various aspects of victimization.

\section{INTRODUCTION}

C'est un plaisir et un honneur de pouvoir contribuer à ce numéro spécial de la revue Criminologie dédié à mon cher ami et collègue distingué, le $\mathrm{D}^{\mathrm{r}}$ Denis Szabo, à l'occasion de son $65^{\mathrm{e}}$ anniversaire. Je salue la décision ainsi que le choix du thème par le comité de rédaction. Il me semble tout à fait approprié de consacrer ce Festschrift au thème «Criminologie Comparée», car je ne connais aucun criminologue vivant qui a autant fait pour promouvoir cette branche de la science criminologique que le professeur Szabo. En effet, le nom de Denis Szabo est devenu presque synonyme des recherches transculturelles visant à acquérir une meilleure connaissance du phénomène criminel par l'observation, la mesure et l'analyse comparatives.

Ayant étudié et enseigné la sociologie et la criminologie en milieu francophone, Denis Szabo compte parmi ses moniteurs intellectuels les géants de la sociologie française, tel que Comte et Durkheim. Et, qu'il l'admette ou non, il n'y a à ce sujet aucun doute dans mon esprit, Szabo fut influencé assez profondément par le juriste/sociologue français Gabriel Tarde. Tarde a inspiré beaucoup de criminologues importants. Sa théorie et ses lois de l'imitation sont à l'origine des theories criminologiques qui mettent l'accent sur l'apprentissage, telle que la thérie de l'association différentielle de Sutherland. L'étude de Tarde sur la criminalité comparée a sûrement joué un rôle important dans la décision de Szabo de vouer la plus grande partie de sa vie académique à la cause de la criminologie comparée.

1. Ph.D., Professeur, Ecole de criminologie, Université Simon Fraser, Burnaby, C.-B., V5A 1 S6. 
Si on me demande personnellement d'indiquer les deux livres qui ont le plus influencé ma pensée criminologique, je choisirai, sans hésitation, le livre de Hans von Hentig, Le criminel et sa victime (1948), et celui de Gabriel Tarde, La criminologie comparée (1886). Ce dernier livre, relativement petit, qui ne fut jamais traduit en anglais, a fait sur moi une impression forte et durable. Le concept de "relativité du crime», si bien exposé et illustré par Tarde à l'aide de nombreux exemples, reste toujours, à mon avis, la critique la plus formidable des théories biologiques et des autres théories dispositionnelles des causes du crime. C'est le livre de Tarde et ses arguments lucides qui m'ont convaincu de la supériorité des explications socioculturelles de la criminalite. Ils ont ouvert mes yeux à la valeur, à l'importance et la nécessité de recherches comparatives et transculturelles en criminologie. C'est précisément le champ que Denis Szabo a choisi de développer utilisant son excellent talent de l'organisation et son dynamisme extraordinaire. Le reste, bien entendu, est de l'histoire, et je suis certain que d'autres collaborateurs à ce volume auront beaucoup plus à dire sur son énorme contribution à la criminologie comparée. Le sujet de cet article n'est cependant pas la criminologie comparée, mais la victimologie comparée.

La victimologie comparée est toujours dans son enfance. Certains vont même jusqu'à contester le terme « victimologie comparée " prétendant qu'il n'est pas justifié étant donné le nombre très peu élevé d'études qui peuvent être qualifiées comme étant de véritables études victimologiques comparatives. Quoi qu'il en soit, le but de cet article n'est pas de présenter un résumé ou une synthèse de ces études comparatives, mais d'offrir quelques réflexions personnelles sur la relativité culturelle de la victimisation.

\section{L'HÉGÉMONIE CULTURELLE DE L'OCCIDENT}

Bien que la criminologie semble avoir accepté depuis quelque temps le fait que la définition de ce qui est et de ce qui n'est pas un crime est essentiellement culturelle, donc relative, une croyance bien répandue, particulièrement parmi les victimologues, veut que la victimisation puisse être universellement définie. À l'instar de la pensée criminologique du XIX siècle, beaucoup semblent croire à présent que certaines valeurs, certains standards de comportement, certains modes d'interaction sont naturels, universels, et transcendants ! Si une telle conviction paraissait justifiée au XIF ou XIIF siècle, lorsque la connaissance d'autrui était fort peu développée, elle paraît assez ridicule au $\mathrm{XX}^{\mathrm{e}}$ sieccle à en juger par les connaissances accumulées sur les communautés, les nations et les sociétés qui habitent les cinq continents.

Il est normal pour les gens qui vivent isolés, dans des lieux très lointains, et qui n'ont ni accès au monde extérieur, ni connaissance de celui-ci, ni contact avec lui, de penser que leur territoire géographique est le centre de l'univers. Grâce à nos connaissances assez développées de l'univers, 
nous ne souffrons pas de ce centrisme géographique, mais nous sommes affligés d'un complexe de supériorité culturelle. Ce complexe nous fait croire que les normes, valeurs, idéaux (moraux et autres) de notre culture sont supérieurs à ceux des autres cultures. On pourrait donner de nombreux exemples afin d'illustrer ce sentiment de supériorité culturelle dont nous sommes affliges. En voici quelques-uns.

Si nous pratiquons la monogamie au lieu de la polygamie, la monogamie doit donc être la norme. Si nous croyons que les femmes doivent cacher leurs seins et que les individus des deux sexes doivent couvrir leurs organes sexuels, la nudité doit alors être jugée indécente et anormale. Si nous considérons le sexe comme un mode de procréation et rien d'autre, et si nous jugeons que les pratiques sexuelles avant le mariage sont immorales ou, l'avortement, un péché mortel, il doit en être ainsi partout, et nous faisons de notre mieux pour que ces pratiques soient condamnées dans les autres sociétés et les autres cultures. Si notre système économique et politique est le capitalisme, alors le socialisme et le communisme doivent être la personnification du mal sur la terre.

En somme, on a tendance à voir nos normes, valeurs et standards culturels comme les produits d'une loi naturelle, ayant une validité universelle, les seuls acceptables, et les meilleurs pour la race humaine. S'il en est ainsi, il n'est donc pas difficile de comprendre pourquoi les missionnaires arrivant de l'Europe au Canada furent choqués par les pratiques entièrement différentes des autochtones, qu'ils appelèrent invariablement « sauvages ».

Cette haute opinion que nous avons de notre culture et nos efforts constants et infatigables pour imposer nos valeurs aux autres équivalent à une hégémonie culturelle. Et puisque nous utilisons nos propres normes et nos standards comme une mesure universelle sur laquelle tout comportement devrait être jugé, il est tout à fait naturel que nous ayions tendance à imposer nos propres definitions de «victime» et de «victimisation » aux gens de cultures qui, très souvent, ont des standards et des idéaux tout à fait différents des nôtres.

Quoique j'aie critiqué cette hégémonie culturelle à plusieurs reprises déjà (Fattah, 1984), la décision de consacrer à ce sujet cet article en hommage à Denis Szabo fut prise assez récemment, lorsqu'un incident particulier m'a rappelé encore une fois le zèle missionnaire de plusieurs adhérents de ce qu'on peut appeler « la nouvelle victimologie».

À une réunion internationale sur les victimes organisée par ${ }^{1}$ en Espagne (mai 1993), une participante m'a confié que son agence essaie, entre plusieurs choses, d'interdire la pratique de la circoncision masculine. Bien que

1. ISPAC Intemational Scientific and Professional Advisory Council (ONU). 
j’aie été au courant. depuis quelque temps déjà, des efforts louables (y compris de ceux des Nations Unies) pour abolir la pratique de la l'excision féminine, $c$ 'était la première fois que $\mathrm{j}$ 'apprenais que la circoncision masculine faisait l'objet d'une campagne visant à son interdiction générale. Cette nouvelle était vraiment une surprise car, originaire d'une culture où la circoncision masculine est très répandue, je me rappelle très nettement encore la honte et le sentiment d'infériorité qu'éprouvaient les garçons non circoncis, ainsi que leur désir ardent de l'être le plus tôt possible.

Émettre un jugement sur la đésirabilité ou l'indésirabilité d'une pratique culturelle assez répandue et vouloir imposer ce jugement aux autres soulèvent une question très importante pour la victimologie et la criminologie. Quel jugement de valeur doit déterminer la forme, l'étendue et la gravité de la victimisation? Le jugement de valeur du chercheur, du praticien, de l'activiste politique doit-il prévaloir sur les perceptions, les définitions subjectives et l'expérience vécue des sujets étiquetés comme victimes, souvent contre leur volonté ou leur gré ? Je reviendrai plus tard sur ce point en analysant la catégorie que j'appelle les «victimes crées par la loi» (legally-created victims).

Il est extrêmement rare de voir cette question posée dans les écrits pourtant abondants sur les victimes. David Miers (1989: p. 12), faisant référence à la critique générale adressée par Biderman (1981 : p.794) aux enquêtes de victimisation, a recommandé qu'en construisant les instruments de recherche, on devrait être sensible à la perception que la victime a des événements victimisants, qu'une telle perception doit l'emporter sur celle du chercheur.

Mais les temps ont sûrement changé ! Au cours des années 1960 et 1970, les criminologues étaient fort critiques à l'égard de ceux que Howard Becker (1962) a qualifiés d'entrepreneurs moraux. Les criminologues ont dénoncé ceux qui essayent d'imposer aux autres leurs convictions religieuses et leurs croyances morales et qui pour ce faire préconisent l'emploi de la loi criminelle et les sanctions pénales comme instruments de pression et d'intimidation. Hélas, aujourd'hui en matière de nouvelle victimologie, l'entreprise et le prosélytisme idéologiques sont des formes bien acceptées d'activisme et de plaidoirie.

Les réflexions exprimées dans cet article et les questions sont donc destinées à prévenir les lecteurs contre le danger que présentent ces nouveaux entrepreneurs idéologiques.

\section{QUI EST UNE VICTIME ? QU'EST-CE QUE LA VICTIMISATION ?}

Le crime, le criminel sont des constructions sociales et culturelles. Il en est de même pour les victimes et la victimisation. Le crime ne peut être défini en des termes abstraits, mais seulement en renvoyant à une société ou 
à une culture particulières (Fattah, 1984). Il en est ainsi pour la victimisation car la victimisation, en plus d'être une expérience personnelle et subjective, est avant tout une construction culturelle.

Très peu de valeurs sont universelles, et ce qui est bon pour les uns n'est pas nécessairement bon pour tous. La démocratie est probablement le système idéal ou un système de choix dans les pays où les citoyens ont atteint un niveau de connaissance et de discipline leur permettant d'exercer leurs droits démocratiques. Pour une société vivant encore à l'âge de bronze ou de pierre, elle peut être désastreuse ! La démocratie peut fonctionner au collège ou a l'université; elle ne fonctionne pas au jardin d'enfants !

Du reste, la vie humaine n'a pas la même valeur dans toutes les cultures, comme en témoignent les différences assez considérables dans le taux de suicide (et d'homicide) entre les sociétés. Appliquant nos propres valeurs, nous définissons toujours le suicide comme une autovictimisation, bien que la personne qui met fin à ses jours puisse regarder son acte, et souvent le regarde ainsi, comme une forme ultime de salut, comme un soulagement immédiat et bienvenu. Si le droit à l'autodétermination est accepté, des termes tels que "victime» et « victimisation " sont inappropries dans le contexte. Continuer de les utiliser trahit une attitude arrogante (nous sommes les juges, nous connaissons mieux) et paternaliste (ce n'est pas bon pour vous, vous ne devez pas faire ça). En effet, dans certaines cultures, le suicide n'est pas considéré comme une autovictimisation mais comme un geste de courage, un acte audacieux, ou un moyen par lequel l'individu admet sa culpabilité et accepte sa responsabilité.

Ceci est pour dire qu'il est tout à faire nécessaire, avant que la victimologie comparée puisse se développer et s'épanouir, de chercher la réponse à travers des études empiriques transculturelles, à plusieurs questions fondamentales dont voici quelques-unes :

- Les notions occidentales de victimisation sont-elles pertinentes pour les peuples de d'autres sociétés et les gens d'autres cultures?

- Dans quelle mesure nos définitions de la victimisation, qui reposent, sur le matérialisme occidental et la morale judéo-chrétienne, s'appliquent-elles aux expériences des membres des sociétés ayant une culture, des traditions, des convictions religieuses et des valeurs morales très différentes des nôtres?

- Qu'a-t-on à gagner en essayant d'imposer nos propres definitions culturelles de "victime» et de «victimisation" aux autres peuples qui ne partagent pas ces définitions?

Les quelques exemples qui suivent, et on peut en donner tant d'autres, ont pour but d'illustrer la variabilité énorme des définitions culturelles de la victimisation. 
- Dans une société d'acquisition, obsédée par les valeurs matérielles et les biens de consommation ou, à tout le moins, qui leur attache beaucoup d'importance, être privé de quelque chose est considéré comme une forme de victimisation sérieuse. Par contre, dans une culture où les valeurs spirituelles dominent, où les gens dédaignent les biens matériels et les activités mondaines, les victimes réelles ne sont pas ceux qui sont privés, mais ceux qui sont esclaves de leur besoin incontrôlable d'acquérir.

- Dans les cultures occidentales, le travail manuel des enfants et le travail dur et prolongé est regardé comme une exploitation, une victimisation, et assez souvent même est punissable par la loi. Mais, dans plusieurs sociétés, le child labour n'est pas seulement une nécessité absolue, c'est aussi un signe infaillible et rassurant d'intégration, de contribution, de participation, d'appartenance et d'acceptation. Les enfants qui, pour une raison ou une autre, ne sont pas mis à contribution souffrent d'un sentiment profond de frustration et de victimisation. Ils se sentent, exclus, ostracisés et sont hantés par le sentiment d'être inutiles et non productifs.

Exploitation et victimisation, comme abus et négligence, sont des conceptions culturelles. Elles ne peuvent être définies ou appliquées sauf en des termes purement culturels, dans le contexte d'une culture bien précise. Dire que la ligne de démarcation entre les conduites acceptables et les conduites abusives est essentiellement culturelle, c'est affirmer ce qui est évident.

Faut-il rappeler qu'il n'y a aucune mesure universelle selon laquelle les comportements humains peuvent être jugés normaux ou abusifs, soigneux ou négligents, acceptables ou non acceptables. Qu'il s'agisse de mauvais traitements à l'égard des enfants, entre époux, ou à l'endroit des gens âgés, la définition de ce qui constitue un «mauvais traitement», exige de se référer aux normes culturelles de la société qu'on étudie. Et que l'accent soit mis sur l'abus physique, psychologique, sexuel, matériel ou autre, la décision de qualifier un acte (ou une omission) comme étant ou n'étant pas un abus dépend entièrement des standards culturels en vigueur.

Ce qu'on vient de dire s'applique aussi bien à la définition de la négligence. Les normes quant aux soins varient énormément d'une société à l'autre et d'une culture à l'autre. Et au sein d'une même société, ces normes peuvent varier considérablement d'un groupe ou d'une classe sociale à l'autre. Selon quelles normes devrait-on donc décider si un enfant dit négligé doit demeurer dans sa famille ou être confié à des parents adoptifs ou à une institution? Selon quelles normes devrait-on décider si une personne âgée, vivant seule ou avec d'autres, est négligée et doit donc être placée dans un nursing home? Ces questions ne sont pas superflues. Au contraire, leur 
importance ne peut être sousestimée, car souvent les normes de celui qui doit prendre la décision sont différentes de celles des parents accusés de négligence ou des vieillards qui sont, croit-on, négligés.

La violence elle-même, souvent invoquée pour justifier ou défendre l'argument en faveur de définitions et de catégories universelles de victimisation, comporte un aspect culturel important. Voilà qui explique les différences assez considérables dans la signification de violence et l'énorme difficulté qu'il y a à vouloir donner une définition objective et universelle de sa nature.

Ceux qui s'opposent à la peine capitale y voit une victimisation violente et cruelle, une sorte de meurtre commis de sang-froid. Pour ceux qui l'approuvent, elle n'est pas un acte de victimisation, mais de justice.

Pour une bonne partie de la société, l'avortement est considéré comme une victimisation sérieuse qui prive un être humain de la chance de naître et de vivre. Dans plusieurs sociétés où un nombre important d'individus crèvent de faim, la même pratique est regardée, non comme une victimisation, mais comme un acte de pitié et de charité, comme un geste qui épargne à l'enfant qui n'est pas encore né une vie de misère, de pauvreté, de faim.

Il en est de même pour l'euthanasie. Pour ceux qui s'y opposent, la victimisation ultime, c'est un homicide prémédité. Pour ceux qui y croient, c'est un geste humanitaire, un acte miséricordieux qui met fin à des douleurs et des souffrances intolérables causées par une maladie incurable. Pour les défenseurs de l'euthanasie, la réelle victimisation, c'est laisser souffrir le malade qui n'a aucune chance de se rétablir et lui nier le choix et le moyen d'y mettre fin.

Ces exemples illustrent bien, je crois, la variabilité des définitions de la victimisation au sein d'une même société et entre les cultures. Mais c'est encore le champ de la sexualité qui fournit, le meilleur exemple de la relativité culturelle de la victimisation.

Dans notre société, tout comportement ou toute pratique sexuelle qui ne sont pas conformes à nos règles morales ou qui violent les tabous religieux sont condamnés, et s'ils sont commis sans le consentement du partenaire ou avec un mineur, sont définis comme une source de victimisation assez sérieuse. Pourtant, dans les cultures plus libérales et moins prudes envers la sexualité, le même comportement ne serait pas nécessairement vécu comme une victimisation.

Dans une analyse perspicace des attitudes à l'endroit de la sexualité, Rubin (1984) fait remarquer que les cultures occidentales considèrent, en général le sexe, comme une force négative, dangereuse et destructrice et le traitent toujours avec suspicion. Cela explique, dit-elle, pourquoi le sexe a toujours été soumis à toutes sortes de contrôles, formels et informels, et 
traité d'une manière très punitive. Rubin insiste sur le fait que les lois qui gouvernent la sexualité sont extrêmement sévères et que les sanctions pour violation des interdictions sexuelles sont sans commune mesure avec le dommage éventuel fait à l'individu ou à la société. Selon Rubin, la loi est particulièrement féroce dans son effort pour préserver la frontière entre «l'innocence» de l'enfant et la sexualité de «l'adulte». Elle écrit :

"Rather than recognizing the sexuality of the young, and attempting to provide for it in a caring and responsible manner, our culture denies and punishes erotic interest and activity by anyone under the local age of consent. The amount of law devoted to protecting young people from premature exposure to sexuality is breathtaking " (1984: p. 290).

On peut être d'accord ou non avec ces attitudes et ces contrôles, mais essayer d'imposer nos valeurs puritaines et nos définitions religieuses et culturelles de la victimisation sexuelle à des peuples appartenant à des cultures très différentes de la nôtre, c'est du colonialisme culturel. Il en résulte que des pratiques et des coutumes parfaitement acceptables dans certaines cultures (comme par exemple l'initiation sexuelle des enfants) sont définies comme une molestation sexuelle des enfants! On oublie souvent que l'âge du consentement aux pratiques et aux relations sexuelles, ainsi que l'âge du mariage, aussi bien pour les garçons que pour les filles, dépend entièrement de la culture et n'est pas fixé de façon uniforme. Et, bien que le passage de l'enfance à l'adolescence soit biologiquement déterminé par la puberté, la frontière qui sépare l'adolescence de l'âge adulte n'est ni claire ni nette, et varie énormément selon la culture. Notre société elle-même a prévu des âges différents pour la majorité civile et pénale, pour le consentement aux actes hétérosexuels et aux actes homosexuels, etc.

Mais ce n'est pas seulement l'âge du consentement qui varie d'une culture à l'autre. Les sociétés chrétiennes et musulmanes accordent une très grande valeur à des vertus comme la virginité et la chasteté. Se voir dérober l'une de ces vertus si hautement estimées est donc considéré comme une victimisation particulièrement grave. De telles attitudes, cependant, ne sont nullement universelles, car dans plusieurs cultures les mêmes qualités ne sont pas perçues comme des vertus, mais comme un fardeau dont il faut se débarrasser le plus tôt possible.

Il va de soi qu'il est aisé à qui appartient à une société d'abondance de s'asseoir confortablement dans un fauteuil de prononcer un jugement de valeur sur l'exploitation sexuelle des enfants ou des adolescents en Thaïlande ou aux Philippines. Tout est de savoir si ces jeunes, qui ont quitté des conditions d'existence horribles où régnaient la saletế et une pauvreté abominable et qui étaient obligés d'accomplir pendant de longues heures un travail manuel et pénible, pour connaître le confort relatif des bordels de 
Bangkok ou de Manille, se considèrent, comme des victimes? Ces jeunes qui ne partagent pas nos tabous sexuels et n'ont pas les mêmes interdictions religieuses vis-à-vis de la sexualité, si on leur donne la liberté de choisir entre leur existence antérieure et la vie dans les bordels où ils sont nourris et logés, que choisiront-ils? Sans des recherches empiriques du type qualitatif, il n'est pas facile de répondre à cette question. Définir une exploitation de cette sorte comme victimisation, en l'absence de telles recherches, n'est qu'un jugement de valeur, une évaluation normative. C'est tout simplement l'expression d'une indignation morale. Une autre question se pose : pourquoi cette indignation et les demandes de faire cesser cette exploitation sexuelle ne s'étendent-elles pas à la pauvreté, à la faim, à l'exploitation physique et aux maladies chroniques dont souffrent les autres enfants dans ces mêmes pays. La victimisation sexuelle est-elle plus affreuse que les autres formes de victimisation? Et si oui, pourquoi?

Poser ce genre de problème, remettre en question les définitions occidentales de victimisation, ou dire que l'évaluation et le jugement de l'observateur occidental ne correspondent pas toujours, à la perception, à l'attitude et aux désirs de ceux qui sont étiquetés « victimes », ce n'est pas nier qu'il y ait une victimisation. C'est se demander, encore une fois, selon quelles valeurs morales, quelles normes, et quelles critères devrait-on déterminer les pratiques qu'on qualifie de victimisation?

La relativité de la définition de ce qui est victimisation et le caractère subjectif de l'expérience dite victimisante sont souvent le résultat d'un écart entre les perceptions des sujets, leurs propres definitions et leur évaluation personnelle de la situation et celles de l'observateur externe.

Le criminologue peut définir la privation de liberté et l'incarcération dans une cellule étroite comme une victimisation cruelle, tandis que le prisonnier, convaincu d'avoir eu un procès équitable, peut considérer sa sentence et l'emprisonnement comme une peine juste et bien méritée. En d'autres mots, il rejette l'étiquette « victime».

Une sociologue ou une psychologue féministe peut définir la relation entre la prostituée et son souteneur comme une situation d'exploitation et de victimisation. Ce jugement externe, cependant, n'est pas toujours partagé par la prostituée elle-même. Souvent, elle ne se considère pas comme victime.

L'écolier qui provoque une bagarre avec un de ses camarades et qui finit par saigner du nez ou avoir un oeil au beurre noir peut croire qu'il a perdu le combat, mais il ne se perçoit pas comme victime et ne définit pas ce qui lui est arrivé comme une « victimisation ». La perception et la définition que donnent ses parents ou la maîtresse d'école de l'incident pourraient être exactement contraires. 
Un autre exemple est celui du joueur de hockey qui souffre d'une blessure grave à la suite d'une bousculade. Bien que celle-ci ait été délibérée, le joueur ne se définit pas nécessairement comme une victime et ne considère pas l'incident comme une victimisation. Les définitions qu'en donne le coach. qui a perdu un joueur précieux, et la partie, sont probablement tout à fait différentes de celles qu'en donne le joueur lui-même.

On oublie souvent que certaines personnes ont dû subir des actes assez violents, provoquant des douleurs et souffrances terribles, ou même des infirmités permanentes, sans pour cela se considérer comme victimes, ou la violence qu'elles avaient subie comme une forme de victimisation. Nous pouvons définir ceux qui ont êté blessés au combat des «victimes de guerre». Eux, qui se considèrent comme des héros, peuvent être insultés par l'étiquette. Un autre bon exemple est celui des rites de passage qui, dans plusieurs sociétés, marquent la transition entre l'enfance et l'état adulte. Bien que ces rites soient souvent assez violents et douloureux, ils ne sont pas vécus comme une victimisation. Dans la majorité des cas, ils sont avidement attendus et, malgré la douleur et la violence sont joyeusement supportés. Une expérience très similaire est celle des rites d'initiation des freshmen au collège ou des nouvelles recrues dans l'armée ou dans la marine. Bien que ces rituels d'initiation impliquent, la plupart du temps, des actes de violence, d'humiliation et de dégradation, ceux qui y sont soumis ne se sentent pas victimisés et ne se définissent pas comme victimes.

C'est un fait bien établi que dans plusieurs cultures (et sous-cultures), l'étiquette victime a une connotation péjorative et est rejetée avec véhémence par ceux qui souffrent. Admettre que l'on a été victimisé ou accepter d'être désigné comme une victime peut souvent être jugé incompatible, avec l'image masculine (celle du macho) et les idéaux culturels de bravoure, de courage et d'endurance. Alors que la victimisation peut être une marque d'honneur dans certaines cultures, dans d'autres elle sera perçue comme une expérience dégradante. Et au même moment où les nouveaux victimologues ont tendance à distribuer l'étiquette «victime» de façon très généreuse, on constate une résistance assez marquée parmi ceux à qui on l'accole. Bon nombre de ceux qui ont subi un viol, un assaut ou un abus quelconque préfèrent le terme survivant à celui de victime. Ajoutons que, dans leur rejet de l'étiquette « victime» ils sont en bonne compagnie.

Les exemples que je viens de donner avaient pour but de souligner la relativité culturelle de la victimisation et la variété énorme de ses définitions d'une société à l'autre. Ils avaient aussi pour but de soulever certaines questions importantes et de mettre en évidence l'obstacle majeur que la victimologie comparée doit surmonter. Ils étaient également destinés à montrer à quel point il est difficile (ou impossible) de mesurer la victimisa- 
tion selon les cultures ou de comparer l'incidence ou le taux de victimisation dans des cultures très différentes.

\section{LES VICTIMES CRÉÉES PAR LA LOI}

Nous l'avons dit, en essayant d'imposer nos normes, valeurs et idéaux aux gens de pays et de cultures très différents, nous finissons par inculquer un sens de victimisation à des individus qui, autrement, ne se seraient pas sentis victimisés, et par étiqueter comme victimes des individus qui ne se perçoivent pas tels.

Nous pratiquons un étiquetage du même ordre quand nous adoptons des attitudes et des lois paternalistes. Dans beaucoup de cas régis par ces lois, les définitions légales de «victime» et de «victimisation» contredisent les définitions qu'en donnent ceux que l'on veut protéger. Il en résulte que l'étiquette "victime» est imposée à des gens qui ne se définissent pas en tant que telles et qui, souvent, contestent avec acharnement cette étiquette. Ils ne sont victimes qu'aux yeux de la loi. À leurs propres yeux, ils sont la cible de contraintes injustifiées, d'interdits absurdes, ou de discrimination pure et simple.

Les exemples des victimes crées par la loi sont assez nombreux (voir Fattah, 1991). Un exemple typique est celui du viol statutaire, où le consentement de la jeune fille (qui n'a pas encore atteint l'âge fixé par la loi) n'est pas admis, ce qui fait d'elle obligatoirement une victime séduite par son partenaire. On peut citer, comme autre exemple, les mineurs à qui on interdit, afin de les "protéger», de faire certaines choses que seuls les adultes ont la permission de faire. Selon la loi, les mineurs qui fréquentent les étabiissements où on consomme de l'alcool, les casinos, les pistes de courses, les cinémas pour adultes, etc., sont des «victimes» et ceux qui les laissent entrer dans ces établissements sont des «victimiseurs». Il va sans dire que ni les uns ni les autres ne perçoivent la situation de la même façon que la loi. C'est qu'en réalité le discours de "protection » est souvent un moyen pour camoufler les restrictions imposées à certains groupes et la discrimination exercée contre ces groupes. Quand, par exemple, le Code criminel fixe un âge différent pour consentir aux actes homosexuels et hétérosexuels, il est assez évident, malgré les justifications rhétoriques invoquées, que cet âge plus élevé n'est pas destiné à protéger ceux qui ont des inclinaisons homosexuelles mais à les discriminer. Et comme l'âge du consentement aux actes homosexuels est en général assez avancé (18 ans ou même 21 ans), un mâle consentant âgé de 20 ans ou de 17 ans et demi (selon la juridiction) est arbitrairement défini par la loi comme une «victime» et son partenaire, comme un adulte «corrupteur». 
Les lois véritablement destinées à protéger certains groupes vulnérables ou souvent exposés ignorent même les besoins émotionnels et sexuels des membres de ces groupes. Ce qu'elles font généralement c'est d'imposer, de façon arbitraire et aveugle, des restrictions aux comportements de ceux dont elles estiment qu'ils ont besoin de protection, les empêchant ainsi de faire ce que d'autres sont autorisés à faire (Fattah, 1991, p. 104). Lorsque la loi qualifie de «viol» le coït avec une personne handicapée mentalement sous prétexte qu'elle est incapable de donner un consentement libre et éclairé, ce que la loi fait, en réalité, c'est d'interdire en permanence une vie sexuelle à une personne qu'on veut protéger. Pour empêcher une exploitation ou une victimisation possible, la loi prive la personne de toute possibilité d'avoir une satisfaction sexuelle avec un partenaire. Ce qui était protection devient en fait une véritable victimisation !

Les lois paternalistes sont problématiques, parce qu'elles vont à l'encontre du fameux principe de liberté énoncé par J. S. Mill. Elles soulèvent la question : jusqu'à quel point la loi devrait-elle essayer de protéger les gens contre leur volonté, ou restreindre la liberté de ceux dont l'âge est inférieur à un seuil arbitrairement fixé ?

Le problème n'est pas limité au droit pénal. Un bon exemple de protection civile qui équivaut à une victimisation sérieuse est le système de tutelle. La tutelle est souvent imposée aux gens âgés qui sont assez facilement, et souvent rapidement, jugés incapables de gérer leurs affaires. C'est une décision très grave, puisque dans beaucoup de juridictions, la tutelle est l'équivalent de ce qu'on appelait autrefois en France la «mort civile». Les conséquences pour la personne âgée qui se trouve subitement dépourvue de tout pouvoir décisionnel peuvent être énormes. La tutelle, c'est évident, est censée la protéger contre une exploitation potentielle. Ironiquement, ce que la loi fait en pratique, c'est de victimiser la personne afin de l'empêcher de devenir victime. En d'autres mots, les individus en tutelle sont, dans les faits, victimisés pour prévenir une victimisation éventuelle!

Il y a bien d'autres exemples où la loi victimise littéralement les gens parce qu'elle juge que cette victimisation est dans leur intêrêt. Ceux qui souffrent d'une maladie douloureuse et sans espoir de guérison et qui veulent mettre fin à leurs jours, mais ont besoin d'aide pour ce faire, sont obligés de souffrir parce que la loi, qui veut les "protéger» continue à définir l'euthanasie, ainsi que l'aide au suicide, comme des délits. 


\section{LA RELATIVITÉ CULTURELLE DES EFFETS DE LA VICTIMISATION}

La relativité culturelle de la victimisation et la subjectivité de toute expérience victimisante signifient que la victimisation, quelle que soit sa nature, n'a pas, et ne peut avoir, les mêmes effets sur tous ceux qui la subissent. Les effets et les conséquences de n'importe quel type de victimisation varient considérablement d'une société à l'autre et d'une culture à l'autre. Ils peuvent aussi varier de façon assez significative d'un groupe et d'un individu à l'autre, au sein de la même société. On soutient souvent, par exemple, que les effets de la victimisation sont plus plus traumatisants pour les vieux que pour les jeunes (Fattah et Sacco, 1989).

Voilà qui constitue, sans doute, un champ fertile et prometteur pour les recherches en victimologie comparée. Malheureusement, la victimologie comparée, comme la criminologie comparée, est dominée par les études de type quantitatif (par exemple, The International Crime Survey, Van Dijk, Killias, and Mayhew, 1989). De plus, la croissance phénoménale dans les services aux victimes et les programmes d'aide aux victimes n'a pas été précédée ou ne s'est pas accompagnée de recherches qualitatives, transculturelles, visant à donner une meilleure compréthension des effets différentiels de la victimisation, des mécanismes différents du coping, du rôle joué par les attitudes culturelles et sociales, soit en accélérant, soit en ralentissant le processus de récupération.

En l'absence de ce type de recherche en victimologie comparée, les remarques qui suivent doivent être considérées comme des hypothèses encore à vérifier plutôt que des faits établis. Mais, malgré l'absence de recherches solides dans ce domaine, il semble logique, voire évident, que les effets de la victimisation ne sont pas les mêmes partout. Dans certaines sociétés, les effets négatifs sont amplifiés, tandis qu'ailleurs, seuls les effets positifs sont soulignés. Il est aussi permis de penser que les blessures physiques n'ont pas partout la même importance et que celle-ci dépende d'un certain nombre de variables.

Nous savons aussi que les blessures psychologiques et les traumatismes mentaux guérissent plus facilement et plus rapidement dans certaines cultures que dans d'autres. Ce qu'il faut savoir, ce sont les caractéristiques des cultures qui facilitent le processus de guérison et celles des cultures qui retardent le rétablissement de la victime. Quel rôle jouent, par exemple, les notions religieuses de «kismet», «destin», «fatalité »? Atténuent-elles le traumatisme en rendant les victimes plus résignées à leur sort de victime? Quel effet l'institution culturelle de la «vendetta» a-t-elle sur le processus de guérison? Les effets traumatiques disparaissent-ils aussitôt la vengeance accomplie? Quel rôle le network social joue-t-il? Nous savons 
tous que le network est plus développé, plus fort et plus cohésif dans certaines sociétés que dans d'autres. Pourquoi les mécanismes et les stratégies du coping sont-ils mieux développés dans certaines cultures et dans certaines sociétés que dans d'autres?

On ne saurait nier que les attitudes et les structures sociales jouent un rôle important dans le processus de guérison des victimes. Peut-on douter, que le rétablissement est généralement plus rapide et plus complet dans les cultures où la victimisation n'est pas prise trop au sérieux, où les effets sont minimisés, même trivialisés? Si cela est vrai, et de prima facie il semble qu'il en soit ainsi, alors les efforts récents des sociétés occidentales pour apporter une aide professionnelle aux victimes pourraient avoir des effets inattendus. De même, les efforts du victim lobby pour sensibiliser le public au triste sort des victimes pourraient avoir aussi des conséquences indésirables. Il semble plausible, par exemple, que l'aggravation de la victimisation et de ses conséquences freine la guérison de la victime et rende son rétablissement plus difficile. Le fait de répéter que les effets de la victimisation (sexuelle ou autre) sont traumatisants, prolongés, etc., peut agir comme un rappel constant de la difficulté du rétablissement, lequel est compliqué et plutôt long, que le retour à l'état antérieur à la victimisation n'est pas facile. Ce rappel peut devenir une self-fulfilling prophétie. Cette stratégie, en plus, semble être à l'opposé de celle utilisée par les médecins et par les psychiatres lorsqu'ils traitent des maladies physiques ou psychiques. Dans ces cas, ils prennent une position rassurante, adoptent une attitude pleine d'espoir et essayent de transmettre une perspective d'avenir optimiste.

L'aide professionnelle apportée aux victimes, même si elle est généralement faite avec les meilleures intentions du monde, peut aussi avoir un effet contraire et donner aux victimes l'impression qu'il leur sera impossible de récupérer par elles-mêmes et que, pour se rétablir, elles auront besoin absolument d'une aide professionnelle.

Un autre domaine qui offre d'excellentes perspectives à la recherche comparative transculturelle en victimologie est celui de la réaction sociale a la victimisation et de son effet ou non sur le rétablissement de la victime. $\grave{A}$ l'heure actuelle, nous n'avons pas la moindre idée si les victimes récupèrent mieux ou moins bien dans des systèmes de justice rétributive que dans des systèmes de justice restaurative. La médiation, la réconciliation, la restitution, le dédommagement, ont-ils ou non un effet plus positif sur le rétablissement de la victime que les sanctions punitives? La guérison estelle conditionnelle à la satisfaction des instincts vindicatifs de la victime par l'imposition d'une peine infligeant douleur et souffrance au malfaiteur? 


\section{CONCLUSION}

Bien que la victimologie comparée soit encore à l'état embryonnaire, les possibilités qu'elle offre à la recherche transculturelle sont excellentes et les perspectives d'avenir semblent très prometteuses. C'est un domaine où l'anthropologie et l'ethnologie peuvent apporter une contribution assez significative. Jusqu'à maintenant, la tendance semble privilégier, comme en criminologie comparée, les études quantitatives, bien que le besoin réel soit une recherche qualitative portant sur différents aspects du phénomène de la victimisation. Certains de ces aspects ont été abordés dans le passé, mais le but de ces recherches était de donner quelques exemples et de proposer un inventaire des recherches possibles en victimologie comparée.

Il demeure, bien entendu, plusieurs obstacles à la recherche transculturelle en victimologie, ainsi qu'une gamme de problèmes conceptuels et méthodologiques qui doivent être résolus pour que, dans ce domaine la recherche donne les résultats escomptés.

Un de ceux-ci est la relativité culturelle de la victimisation et la nature très subjective de l'expérience d'être victime. La relativité culturelle du crime n'a pas été un obstacle insurmontable en criminologie comparée; il n'y a donc pas de raison à ce que la relativité culturelle de la victimisation entrave les progrès de la victimologie comparée. La relativité culturelle signifie tout simplement que des recherches qualitatives menées par des équipes multi-disciplinaires, d'anthropologues et d'ethnologues sont plus susceptibles de faire avancer notre connaissance du phénomène de la victimisation qu'une simple comptabilité des incidents de victimisation.

\section{BIBLIOGRAPHIE}

BECKER, H. (1962), Outsiders : Studies in the Sociology of Deviance, New York, Free Press of Glencoe.

BIDERMAN, A.D. (1981), «Sources of Data for Victimology », Journal of Criminal Law and Criminology. Vol. 72, p. 789.

FATTAH, E.A. (1984), Introduction to Criminology - A Study Guide. Vancouver : Simon Fraser University, Centre for Distance Education.

FATTAH, E.A. (1986), « On Some Visible and Hidden Dangers of the Victim Movement ", in E.A. FATTAH (ed.), From Crime Policy to Victim Policy - Reorienting the Justice System. London : MacMillan.

FATTAH, E.A. et V.F. SACCO (1989). Crime and Victimization of the Elderly, New York : Springer Verlag.

FATTAH, E.A. (1989), The Plight of Crime Victims in Mordern Society, London : MacMillan. 
FATTAH, E.A. (1989), "Victims and Victimology : The Facts and the Rhetoric», International Review of Victimology. Vol. 1, $\mathrm{n}^{\mathrm{O}} 1, \mathrm{p} .43-66$.

FATTAH, E.A. (1991), Understanding Criminal Victimization, Scarborough, Ontario, Prentice Hall Canada.

FATTAH, E.A. (1992), Towards a Critical Victimology, London : MacMillan.

FATTAH, E.A. (1993), «The Rational Choice/Opportunity Perspectives as a Vehicle for Integrating Criminological and Victimological Theories », Advances in Criminological Theories, Vol. 4, p. 225-258.

FATTAH, E.A. (1993), The Criminalization of Social Problems : Child Abuses as a Case Study, In Press.

HENTIG, H. VON (1948). The Criminal and his Victims, New Haven : Yale University Press.

MIERS, D. (1989), Positivist Victimology : A Critique. International Review of Victimology, Vol. 1, no 1, p. 3-22.

RUBIN, G. (1984), «Thinking Sex : Notes for a Radical Theory of the Politics of Sexuality , in Carole S. Vance (éd.), Pleasures and Danger : Exploring Female Sexuality, London : Routledge and Kegan Paul, p. 267-319.

TARDE, G. (1886), La criminalité comparée, Paris.

VAN DIJK, J.J., P. MAYHEW, et KILLIAS, M. (1990), Experiences of Crime Across the World - Key Findings of the 1989 International Crime Survey, Deventer : Kluwer Law and Taxation Publishers. 